\title{
Proceeding
}

Supplementary Issue: Autumn Conferences of Sports Science. Costa Blanca Sports Science Events, 18-19 December 2020. Alicante, Spain.

\section{Definition of physical-dynamic parameters in circular kick in Muay Thai through latest generation inertial sensors with a critical review of the literature}

\author{
MICHELA SOCCI ${ }^{1}$, CIRO HOSSEINI VARDE'I ${ }^{1}$, MARCO GIOVANNELLI ${ }^{1}$, ANTONIO CEJUDO ${ }^{2}$, \\ FRANCESCA D'ELIA D'ELIA ${ }^{3}$, ALBERTO CRUCIANI ${ }^{4}$, RICCARDO IZZO1,2 \\ ${ }^{1}$ Department of Biomolecular Sciences, School of Health and Sport Science, University of Urbino Carlo Bo, \\ Urbino, Italy \\ ${ }^{2}$ Department of Physical Activity and Sports, Faculty of Sports Sciences, University of Murcia, Spain \\ 3 University of Salerno, Italy \\ ${ }^{4}$ Development Engineering Department, Stats Perform, K-Sport Universal, Italy
}

\begin{abstract}
In recent years, the study of movement analysis with the application of dedicated advanced technologies, such as inertial technologies, has also become essential in scientific research of sports disciplines such as martial arts and combat sports, to better define the real dynamics of technical components in order to adapt the training design. A great number of studies in the literature have focused on the kinematics of circular kick in combat sport and martial arts but a relatively small number have focused on the kinetic and kinematic analysis of Muay Thai and especially its circular kick technique. This study aims to highlight instrumentally the quality of circular kick gesture and through which segmental motor dynamics it achieves its greatest effectiveness, evaluating the accelerations and execution times of the different body segments involved in technical execution itself obviously in a performative sense. It is important to note that the inertial data shows that both knee and ankle have an increasing linear velocity greater than the hip in the preparation and execution phase, while for the angular velocity in the execution phase it is the hip that has higher acceleration than the knee and ankle. These results, therefore, highlight the basic importance of intra-articular and intramuscular coordination processes that allow or not linearity and fluidity of movements that are the basis to obtain maximum intersegmental performance in order to deliver maximum acceleration in the apical point of the shot and thus have the maximum effectiveness of hit and therefore the optimal performance.
\end{abstract}

Keywords: Muay Thai; Circular kick; Velocity; Acceleration; Inertial sensors.

Cite this article as:

Varde'i, C.H., Giovannelli, M., Cejudo, A., D'Elia, F., Cruciani, A., \& Izzo, R. (2021). Definition of physical-dynamic parameters in circular kick in Muay Thai through latest generation inertial sensors with a critical review of the literature. Journal of Human Sport and Exercise, 16(2proc), S319-S334. doi:https://doi.org/10.14198/ihse.2021.16.Proc2.17

Corresponding author. Department of Biomolecular Sciences, School of Health and Sport Science, University of Urbino Carlo Bo, Urbino, Italy. https://orcid.org/0000-0002-7530-512X

E-mail: izzore@uniurb.it

Abstract submitted to: Autumn Conferences of Sports Science. Costa Blanca Sports Science Events, 18-19 December 2020. Alicante, Spain.

JOURNAL OF HUMAN SPORT \& EXERCISE ISSN 1988-5202

(C) Faculty of Education. University of Alicante

doi:10.14198/jhse.2021.16.Proc2.17 


\section{INTRODUCTION}

Muay Thai (MT) is a martial art, combat sport, and ancient Thai wrestling technique, it is also known as the "science of the eight arts" because it allows the two contenders facing each other to use combinations of punches, kicks, elbows, and knees, thus using eight parts of the body as points of contact compared to the two in boxing (Turner, 2009). The scientific study of Muay Thai (MT) is young and not very consistent in the literature, such a lack of scientific data clearly represents an obstacle in the optimal preparation of this discipline which, like others, bases its planning substantially on empirical, experiential data that have high margin of subjectivity and therefore often also of error. The first analysis on the kinetics and kinematics of kicks in MT was conducted by Sidthilaw (1997) by analyzing a circular kick through an accelerometer. Subsequently several studies on the kinematics of circular kick in MT conducted by Young (1991), Kim et al. (2010), Aaron J (2015) and Chinnasse C. et al. (2017) have greatly expanded knowledge in this area. In MT, basically three categories of techniques are used such as punches, kicks, and knockdowns, this is called "combination of techniques" (Siesto, 2007). For the purpose of an adequate analysis of the sports performance, it is essential to highlight elements of the performance model (Izzo R., 2011), such as execution times, the biomechanical structure, the energy metabolisms involved, as well as of course the technique and some strategy notes. Combat sports are physiologically definable as hybrids, which means that they involve all three energy systems to varying degrees and always require an optimal combination of various neuromuscular and metabolic abilities including strength, power, aerobic capacity, and endurance. From a metabolic point of view, MT can be defined as an intermittent sport with a high commitment, with phases of maximum or supra-maximal intensity spaced by short recoveries and with great involvement of both aerobic metabolism and anaerobic glycolysis (Crisafulli et al, 2009). Strength and its derivatives, fast, resistant, and explosive characterize a MT athlete and is crucial during combat because a large number of skills depend directly on it. Ring sports are sports for the most part of a "ballistic" nature; both the cardiovascular system, the osteo-articular and muscular system are put to a severe test, and this is also the reason why, in our opinion, it's crucial to work carefully in the preparation of the athlete with truthful, controllable, possibly detected parameters in "LIVE" to optimize and direct the execution of the different series and repetitions of the exercises during the work (Altavilla et al., 2018, D'Isanto et al., 2019).

\section{Types of kicks}

Between the many types of kicks, we have studied the medium circular kick, or "middle kick" (Figure 1), it is performed by moving the leg in a semicircular or circular trajectory by striking with the ankle or tibia. It can also be done in reverse with the heel. Usually, you aim at the opponent's face or torso rather than at the legs. The circular kick develops considerable power. A variant of this technique is the whipped kick, faster and with a less marked trajectory, with less power but still useful if used at the right time (Bos et al, 1993, Nakayama, 1994, Chinnasse et al, 2017).

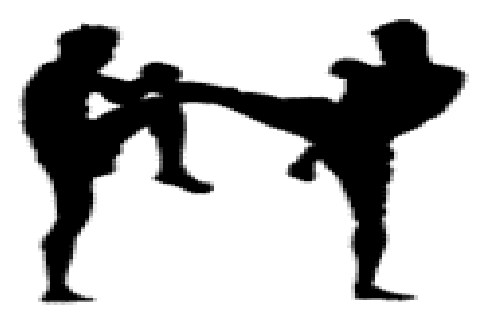

Figure 1. "Middle Kick" from: http://getdrawings.com/ 


\section{State of the art}

Mohamad et al. (2017) in their systematic review "sports science-based research on the sport of MT: a review of the literature", found that their systematic study of the combat sports literature did not produce a satisfactory number of scientific articles for regarding MT. Such a lack of scientific data could represent an obstacle in the optimal preparation of this discipline which, like others, bases its planning exclusively on empirical, experiential data that brings with it a high margin of subjectivity and therefore often of error (Kim et al., 2010, Izzo et al., 2018,). It is easy to think that a more careful analysis of the different performative variables would lead to a higher and more scientific elaboration of the work project. Out of a total of 286 articles, (De Cesari, 2009, Mohamad et al., 2016) only eight (8/286 - 2.8\%) analyzed the objective measurement systems of sports performance in combat sports. This underlines that the application of technology for movement analysis in these disciplines is little studied at a scientific level and in fact shows a considerable delay in the application of the same in a more systematic way to obtain a work system that is certainly updated, appropriate and performing. Worsey et al. (2018) carried out a review of the literature for the analysis of the performance of combat sports athletes, which summarizes the history of use of combat sports sensors specifically. Having said this, it should be emphasized that the first analysis on the kinetics and kinematics of kicks in MT was conducted by Sidthilaw (1997) by analyzing a circular kick using an accelerometer placed inside a bag (hitter). In addition to defining a peak force $(14,000 \mathrm{~N})$ for a circular MT kick, this study analyzed the kinetics and kinematics of three height levels of MT kick, highlighting that middle kick generates the maximum power. During the study it was observed that the linear speed of the circular kick reached the maximum speed $(7.1 \mathrm{~m} / \mathrm{s})$ at 0.48 seconds before impact. A further study by Aaron J. (2015), also related to kinematics, determined the factors most closely related to a high-speed circular kick, in which it was hypothesized and confirmed that the speed during the execution phase before impact is the most influential factor. The three-dimensional analyzes were obtained with a camera system that uses an infrared frequency range by recording the coordinates of the markers. Chinnasse C. et al. (2017), examined the kinematics of the lower limb during the circular kick in MT, both of the dominant and non-dominant leg, with beginners. (Motion Analysis System [Vicon T10s, Oxford Metrics, UK], based on Plug-in-Gate Marker Set, with 29 reflective markers affixed to participants. Colin J. Garavan and Mark GL Sayers (2017), were the first to verify differences in leg kinematics in circular kick performed by experts in MT, Karate and Taekwondo. Several authors such as Roberts et al. (1994) studied the dynamics of the kicking leg, analysing linear velocities of the foot and angular velocities of the hip and knee, carrying out research on the kinetics and kinematics of kicks with the aim of identifying the effectiveness of techniques in the various martial arts. The study of the speed of the gesture, both linear, of some parts of the body, and angular of the joints considered to be the most interesting, can give an indication of the performance of the gesture (Witte K. et al., 2007, Landeo, 2007). In Taekwondo the study by Soresen H. et al. (1996) instead provided information on the timing of the thigh-leg segment of the kicking limb. The tools used were a high-speed video camera $(200 \mathrm{~Hz})$ and an electromyograph. The study showed that during the execution of the kick the leg intervenes after the action of the thigh and that it is the rapid action of the knee that slows the flexion of the hip.

\section{MEANS AND METHOD}

Among the four offensive skills of Muay Thai, the circular kick is the most dangerous weapon with which to hit the opponent (Young, 1991) and can be divided kinematically into several essential components: turning the hips, turning the support foot, swing the arms and strike with the lower tibia or instep (Young, 1991). The circular kick is the most used athletic gesture in martial arts matches due to its usefulness in both attack and defense, its short execution and high probability of scoring (Kim et al. 2010). The circular kick in its three executions generates multiple levels of force for the three main targets, such as, the thighs, the trunk, and the head (low kick, middle kick, high kick) and the athlete to get more strength on impact, must take into 
consideration both the four essential components (preparation-execution-impact-return) and the linear and angular velocity of the kicking leg (Del Vecchio et al., 2015). Although we are aware of the statistically insignificant number of the sample, we would like to underline that the work is highlighted as a pilot application of the latest generation inertial tool to evaluate its effectiveness and, in the positive case, to extend the application to a larger sample (Mathie et al., 2004, Montoye et al., 1996, Silva et al., 2011). The primary purpose of this work is therefore to acquire specific data on the physical characteristics of circular football in MT to assess whether the acquired parameters prove to be significant, however, not yet clearly defined in the literature, for the revision of the specific model of sport, and for the construction of the training work more closely related to the real performance indices of the sport under investigation (Altavilla et al., 2019a, b, Raiola et al., 2020). Define and analyze the acceleration of the hip, knee, and ankle during the execution of the attack with the medium circular kick (middle kick). We consider it useful to remember some points of the studied technique referring to its biomechanics:

- Preparation phase; The body must lean forward noticeably, while performing a semi-twist in the opposite direction to that of the kick, for good balance and correct retraction of the striking foot. The starting position is very important in the execution of the technique: it must be constituted in such a way that the body is arranged in a correct sagittal split and the heels each lie on the perpendicular to the vertical line that passes through the most lateral point of the pelvis from it. side, while the feet will be equal to the width of the pelvis. The trunk naturally orients itself in a semi-frontal position in relation to the opponent's location in space. It is necessary to have the foresight to look towards the opponent and keep it on him throughout the execution of the blow. This not only for tactical reasons to control the opponent's actions, but also to avoid rotational movements at the level of the column that could cause dispersion of kinetic energy. Both the front and rear legs must be semi-bent (Park et al., 1989, Camomilla et al., 2018). From the on-guard position, the weight of the body is moved to the front limb by simultaneously rotating the support foot counterclockwise, this allows us to rotate the pelvis and the trunk in the same direction, so as to rotate the hip and simultaneously extend the kicking leg. The lower limbs must be in sagittal divarication. This trick is done to give greater power to the circular kick, so it may be useful to project the pelvis in a direction that recalls the trajectory of the kick itself (the trajectory of the part of the body that strikes). To do this, it is necessary that one limb is advanced with respect to the other, so that the extension of the rear leg (which is semi-bent part) causes a movement of the pelvis towards the front.

- Execution phase, When the rear leg has performed the deadlift and has started to move towards the target, the weight of the body, which was previously partly distributed on the rear leg, must be transferred entirely to the front leg, the supporting leg. For this to happen, the trunk must perform a forward movement, that is, towards the opponent. This is the internal rotation (intra rotation) of the pelvis (contraction of the oblique abdominal muscles). This is the movement that contributes most significantly to giving the circular trajectory characterizing the shot. However, this movement must coordinate very accurately with the other elements that intrinsically make up the entire blow, because if this were not the case, energy dispersions would occur, the rotation of the pelvis must begin during the leg extension movement and must end at the moment impact with the target to simultaneously express both stable balance and maximum power transfer (Roetenberg, 2006, Zyona et al., 2012). Next, the previously bent kicking leg must extend so that it reaches the target almost fully straight. It will not fully extend before reaching the target because if this is done the power generated by the quadriceps femoris will be exhausted by the action of the antagonists (Silva et al., 2011).

- Return Phase; The movement of rotation of the pelvis and of adduction and extra-rotation of the thigh to return to the starting position in which the limb that was previously posterior here becomes anterior and vice versa. Then the kicking leg can be lowered to regain contact with the ground in the onguard position. 
The instrumentation used was K-Track (K-Sport Universal, Stats Perform, Italy), whose data were processed through the dedicated software of the same company, K-Fitness. The latest generation K-Track inertial sensor with $4000 \mathrm{~Hz}$ potential is equipped with an accelerometer, a gyroscope, and a 3D magnetometer, integrated and have been used at a calibration of $100 \mathrm{~Hz}$ for better coordination between the instruments. The IMU, given its weight of 25 grams and very small measures (a USB memory stick) did not affect the athlete's movements in the least. For our research, only the accelerometer and the triaxial gyroscope were used as the magnetometer, calculating the orientation and direction of the subject, was not used due to its directional function which is irrelevant for the study in question. The device was worn, in its various locations (ankle, knee, hip), using a belt, a knee brace and an anklet that were chosen for the best stabilization and safety of the instrument and the athlete. The study took place in a gym with the ground covered with an approved $4 \mathrm{~cm}$ thick tatami. The first trick was to place some tape on the bag to be hit at a height proportional to the height of the athlete who performed the stroke, that is $1.03 \mathrm{~m}$, measured from the ground. As for clothing, the subject was barefoot, as is the case during official competitions and wore MT shorts and a tank top. The subject anthropometric values were detected: height, weight, and length of the lower limbs' length. Before the test, the athlete was informed of the measurement procedures and the complete test. Subsequently, the subject autonomously began a 10-minute warm-up, with free exercises suitable for obtaining a good performance of the gesture requested, specific usefulness exercises for the test and precompetition practice (Di Stefano, 2004, Kyrolainen et al., 2005).

Table 1. Anthropometric values.

\begin{tabular}{lccccc}
\hline Height & Weight & Experience & Age & Gender & Low. Limb Lgth \\
\hline $1.67 \mathrm{~m}$ & $60 \mathrm{Kg}$ & 9 anni & 28 & $\mathrm{~F}$ & $96 \mathrm{~cm}$ \\
\hline
\end{tabular}

The protocol required as a starting position to assume the "guard position", that is, with the kicking leg dominant in the back, the arms high in the defense position and to start the gesture from standing at the start with maximum speed and then after having hit the target, quickly return to the starting position. At the end of the warm-up, seven repetitions of kicks were performed for each joint analyzed: hip, knee, and ankle, then 21 actions. Between one set of kicks and the next, the athlete performed a 2-minute recovery. The test took place with artificial lighting and with the maximum possible silence and with an ambient temperature of the gym of 20 degrees centigrade (Halil et al., 2010).

\section{DATA ANALYSIS AND DISCUSSION}

The data obtained from the inertial sensors in terms of speed, angular velocity, acceleration, and deceleration regarding the execution of the circular kick in the MT were analyzed using the K-Fitness software (K-Sport Universal, ITA). The data were downloaded to a PC from the instrument memory after the tests. The data from the accelerometer and gyroscope, to analyze acceleration and angular velocity during the Muay Thai round kick, were then placed side by side with the video recordings of the technical gesture to better visualize the motor task (Loong et al., 2011, Martorelli, 2013).

\section{Comparative analysis Synchronized video / IMU}

The recorded videos were analyzed to identify the characterizing moments of the football movement. The frames were then extracted from the video with a duration of 7 seconds, taken by a camera with a sampling frequency of $100 \mathrm{~Hz}$. The frame relating to the moment of impact of the foot on the bag was identified as moment 0 , so it was the observation was focused on the previous 100 frames and on the 100 following ones. The dynamics of the analyzed gesture envisaged that the subject during the measurements started from the 
static defense position, with the dominant leg retracted as in the following images. Based on these events, 3 main phases have been identified, summarized in the following Table 3.

Table 2. Stroboscopic succession of the middle kick technique.

\begin{tabular}{|c|c|c|c|c|c|}
\hline $\mathrm{N}^{\circ}$ & Frame & Event & $\mathrm{N}^{\circ}$ & Frame & Event \\
\hline $1^{\circ}$ & $\begin{array}{lll}0 & 0 \\
0 & 0 \\
0 & 0\end{array}$ & $\begin{array}{l}\text { Guard position - Start } \\
\text { of recording }\end{array}$ & $5^{\circ}$ & ace. & $\begin{array}{l}\text { Max body rotation, ankle } \\
\text { flexion }\end{array}$ \\
\hline $2^{\circ}$ & की & Start of loading & $6^{\circ}$ & rin & $\begin{array}{l}\text { Impact with the bag, } \\
\text { Load release }\end{array}$ \\
\hline $3^{\circ}$ & sise. & $\begin{array}{l}\text { Start of execution: } \\
\text { - Deadlift of the } \\
\text { kicking foot } \\
\text { - Body rotation } \\
\text { - Knee flexion } \\
\text { - Ankle extension }\end{array}$ & $7^{\circ}$ & $\frac{1}{2}-60$ & Beginning of the return \\
\hline $4^{\circ}$ & 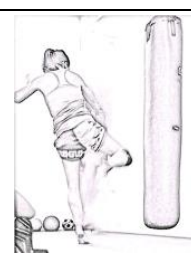 & $\begin{array}{l}\text { Max knee flexion - } \\
\text { start of knee extension }\end{array}$ & $8^{\circ}$ & 泴 & $\begin{array}{l}\text { Footrest on the ground } \\
\text { (starting position) }\end{array}$ \\
\hline
\end{tabular}

Table 3. Three phase of middle kick technique.

\begin{tabular}{lll}
\hline Phase & Start Event & Final Event \\
\hline 1.Preparation Phase & Start of loading & Deadlift of the kicking foot \\
2.Execution Phase & Deadlift of the kicking foot & Impact with the bag \\
3.Return Phase & Impact with the bag & Footrest on the ground \\
\hline
\end{tabular}

\section{Linear acceleration}

As mentioned above, the acceleration data along the three main axes: $X, Y, Z$, according to the convention described above and associated with a point of interest, allowed us to measure the kinematics of the motor task under consideration. From these, the intensity value of the acceleration vector was calculated starting from its three components in each instant of time recorded, for each kick and for each joint studied. To do this, the following formula was used:

$$
A=\sqrt{A c c X^{2}+A c c Y^{2}+A c c Z^{2}}
$$


Where Acc $x, A c c Y$ and $A c c Z$, represent precisely the three vector components of acceleration. For all measurement groups, the point with maximum acceleration has been located, taken as a reference, and set in a new time axis at instant 0 . This peak will represent the instant of impact of the leg on the bag, as also shown by the frames. In processing the data, for each kick performed, a set of 201 measurements was therefore taken into consideration, 100 measurements before the acceleration peak and $100 \mathrm{after}$, for a total of about 2 seconds of test sequence. In this way, for each joint, the average acceleration value over time of the seven kicks performed and the standard deviation were measured. The first expresses a representative value of the measurements, represented by the seven tasks recorded, while the standard deviation quantifies the dispersion of the values of a parameter at a given instant. Both of these indices made it possible to characterize the kick in question. The figures $(1,2,3)$ shown below show the results relating to the average acceleration over time during the movement of the three landmarks of the leg: hip, knee, and ankle.

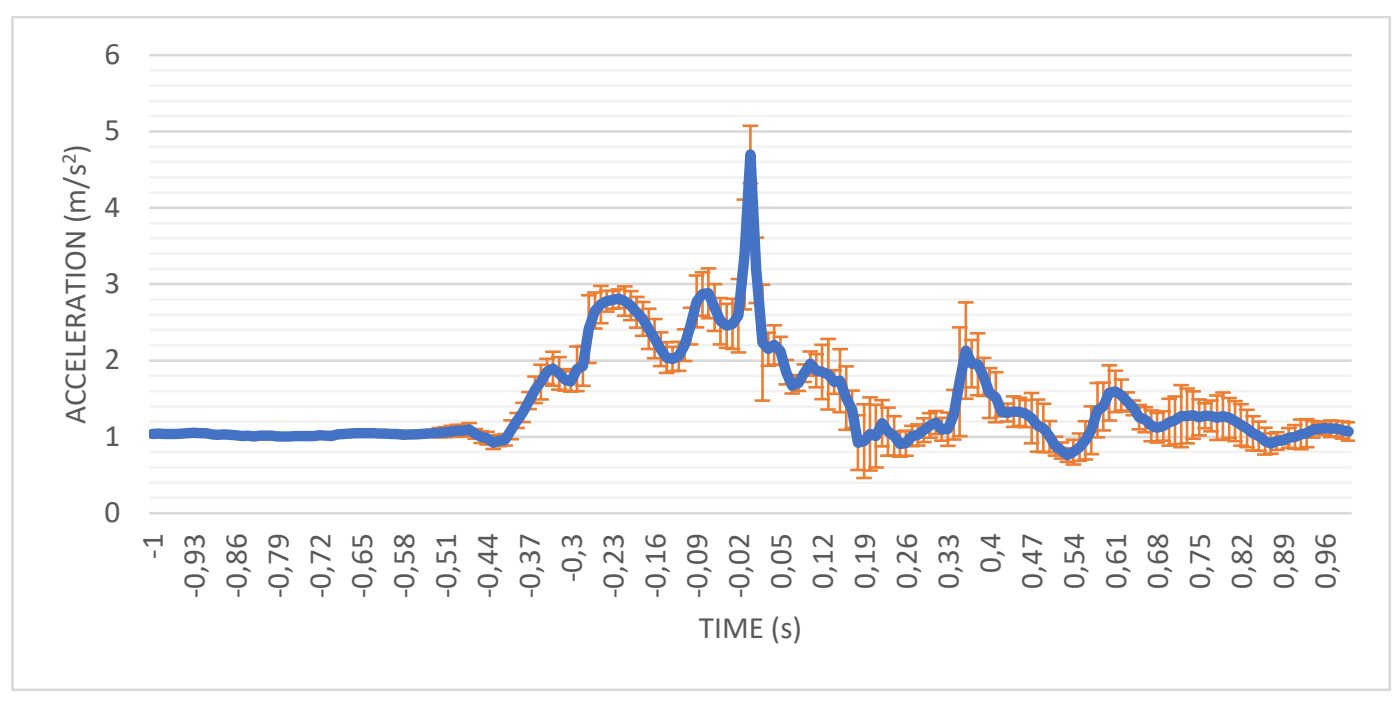

Figure 1. Linear Acceleration - Hip.

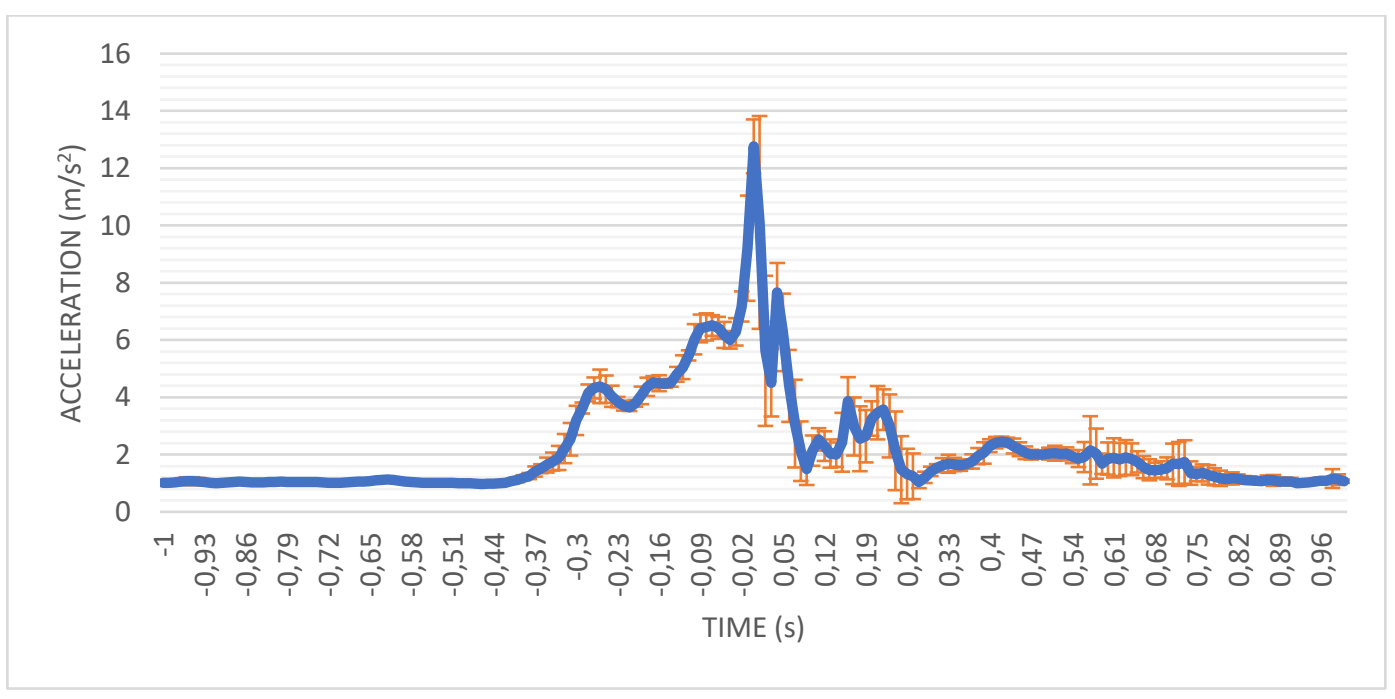

Figure 2. Linear Acceleration - Knee. 


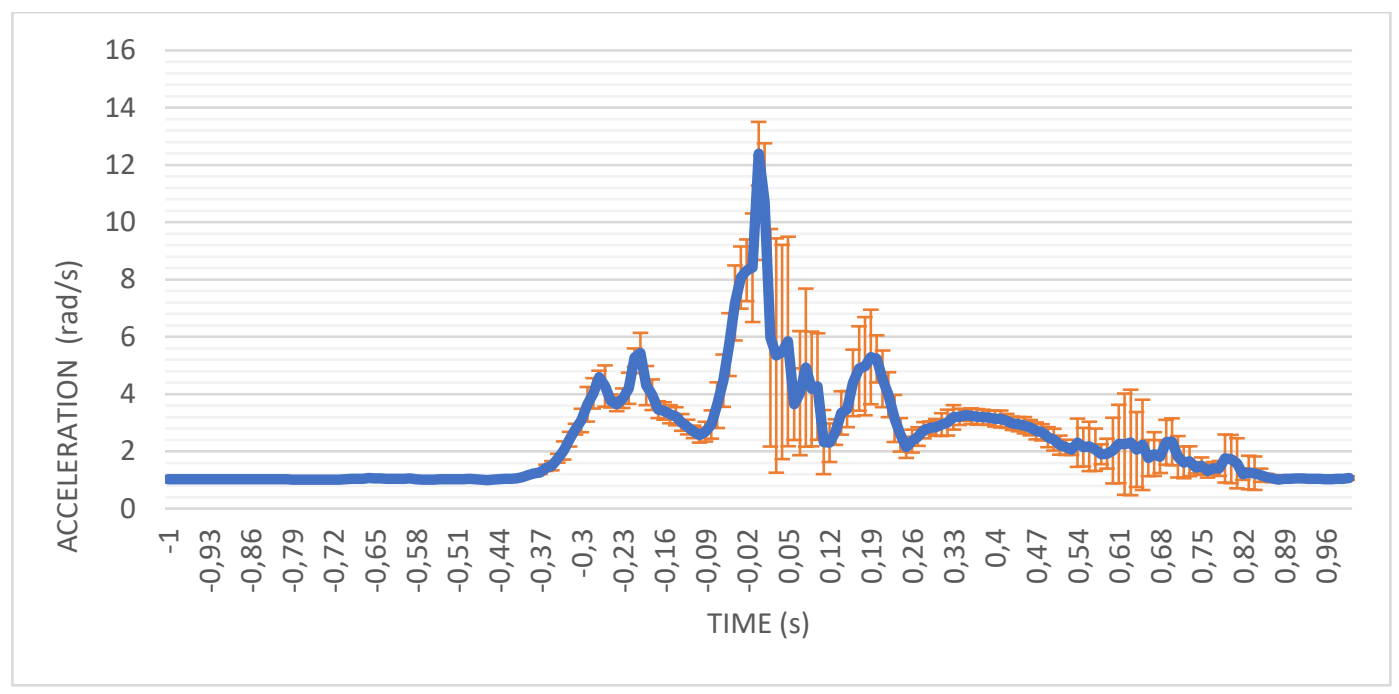

Figure 3. Linear Acceleration -Ankle.

\section{Angular velocity}

Similarly, to linear acceleration, for the angular velocity, which is the ratio between the described angle and the time interval used to describe it ( $\mathrm{rad} / \mathrm{s}$ ), we have identified the intensity of the angular velocity from its components. We calculated the intensity of the angular velocity for each instant of time of all seven kicks and for each joint. To get this value, the following formula was used which is composed of its three vector components:

$$
V=\sqrt{\text { GyroX }^{2}+\text { GyroY }^{2}+\text { GyroZ }^{2}}
$$

Subsequently, the various executions of each joint and each kick were averaged, finding the respective values. The figures below $(4,5,6)$ show the relative results of the average angular speed over time during the movement of the three analyzed points of the leg: hip, knee, and ankle.

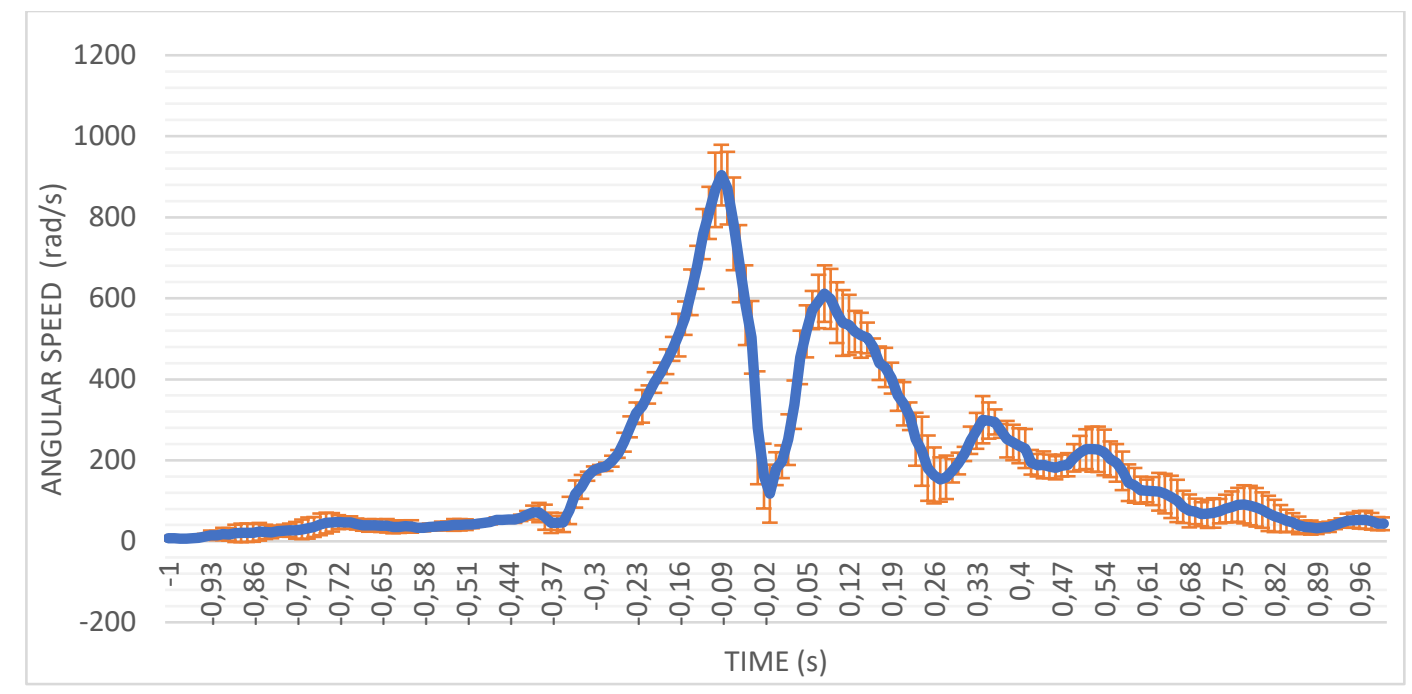

Figure 4. Angular Speed -Hip. 


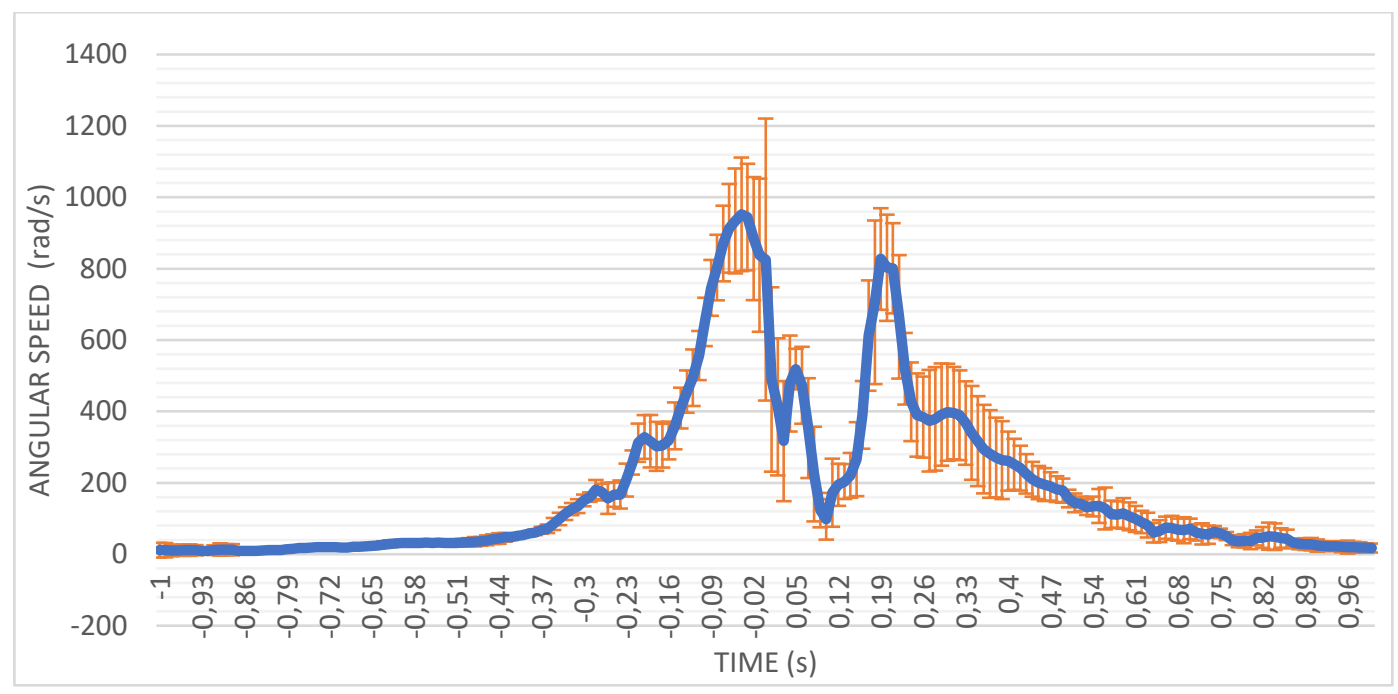

Figure 5. Angular Speed - Knee.

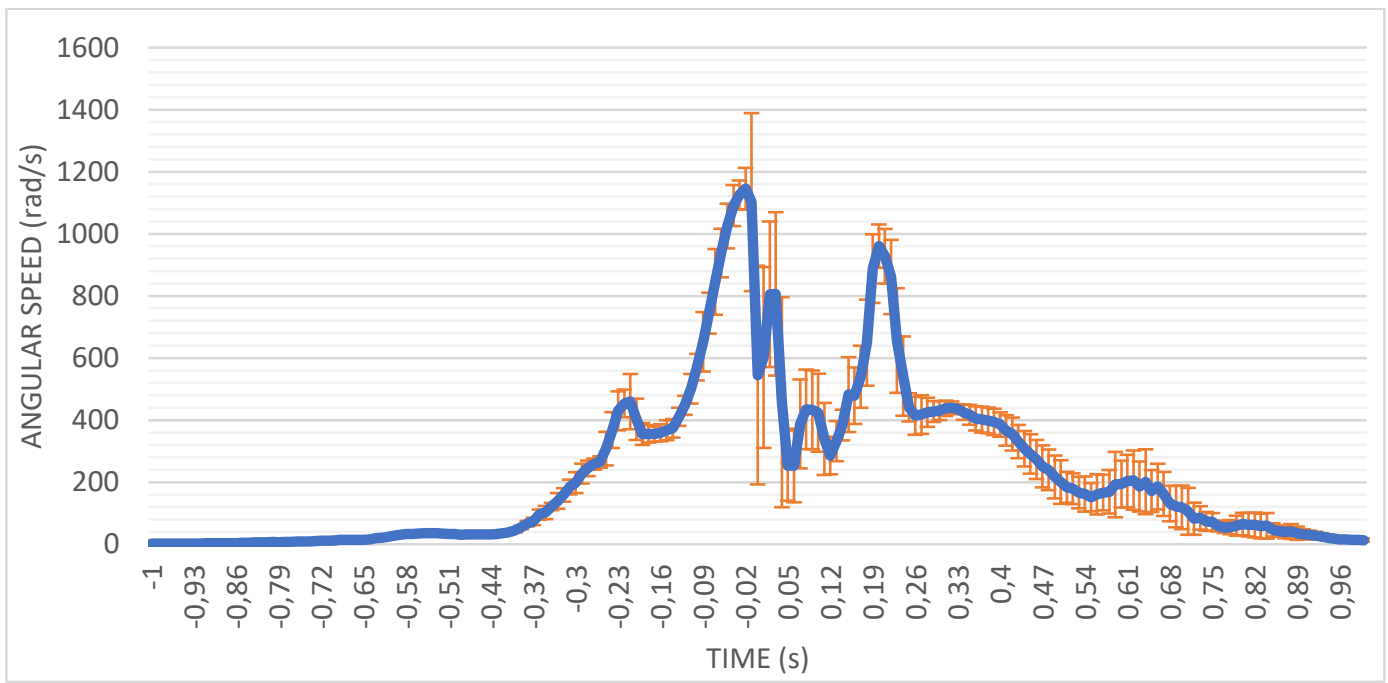

Figure 6. Angular Speed - Ankle.

\section{DISCUSSION}

The figures of the average acceleration for the three anatomical landmarks were aligned with the considerations on the motor task made by observing the frames from the videos. The mean acceleration values, in $\mathrm{m} / \mathrm{s}^{2}$, of the seven kicks for each joint in correspondence with the main events are described in the following Table 4.

Observing the various phases, the following behaviors are noted:

- Preparation phase (I): In this phase, an increase in acceleration can be observed regarding the hip due to the preparation for the execution of the kick itself; the knee and ankle have a point of maximum acceleration at the same instant of time corresponding to the previous load-bearing movement in view of the execution. 
- Execution phase (II): The knee has a tendency of increasing acceleration probably due to the initiation and maintenance of the extension of the leg that precedes contact with the target; observing the peak, it can be seen that the maximum acceleration recorded in the hip has a much lower value than the knee and ankle which, on the contrary, record values that are very similar to each other.

- Return phase (III): The acceleration simply decreases in all joints. In this phase it is easy to see that the standard deviation, an important aspect regarding the dispersion of the data samples, is greater, which means that the kicking leg recall gesture was not performed in a homogeneous and equal manner for all kicks, this aspect is probably given by the fact that the subject performs this phase passively.

Table 4. Time acceleration values.

\begin{tabular}{lcccc}
\hline & Phase I & Phase II & Impact & Phase III \\
\hline Time & $-0.3 \mathrm{~s}$ & $-0.22 \mathrm{~s}$ & $0 \mathrm{~s}$ & $0.46 \mathrm{~s}$ \\
Hip $\left(\mathrm{m} / \mathbf{s}^{2}\right)$ & 1.72 & 2.81 & 4.7 & 1.3 \\
Knee $\left(\mathrm{m} / \mathbf{s}^{2}\right)$ & 3.19 & 3.71 & 12.76 & 2.06 \\
Ankle $\left(\mathrm{m} / \mathbf{s}^{2}\right)$ & 3.08 & 4.2 & 12.39 & 2.83 \\
\hline
\end{tabular}

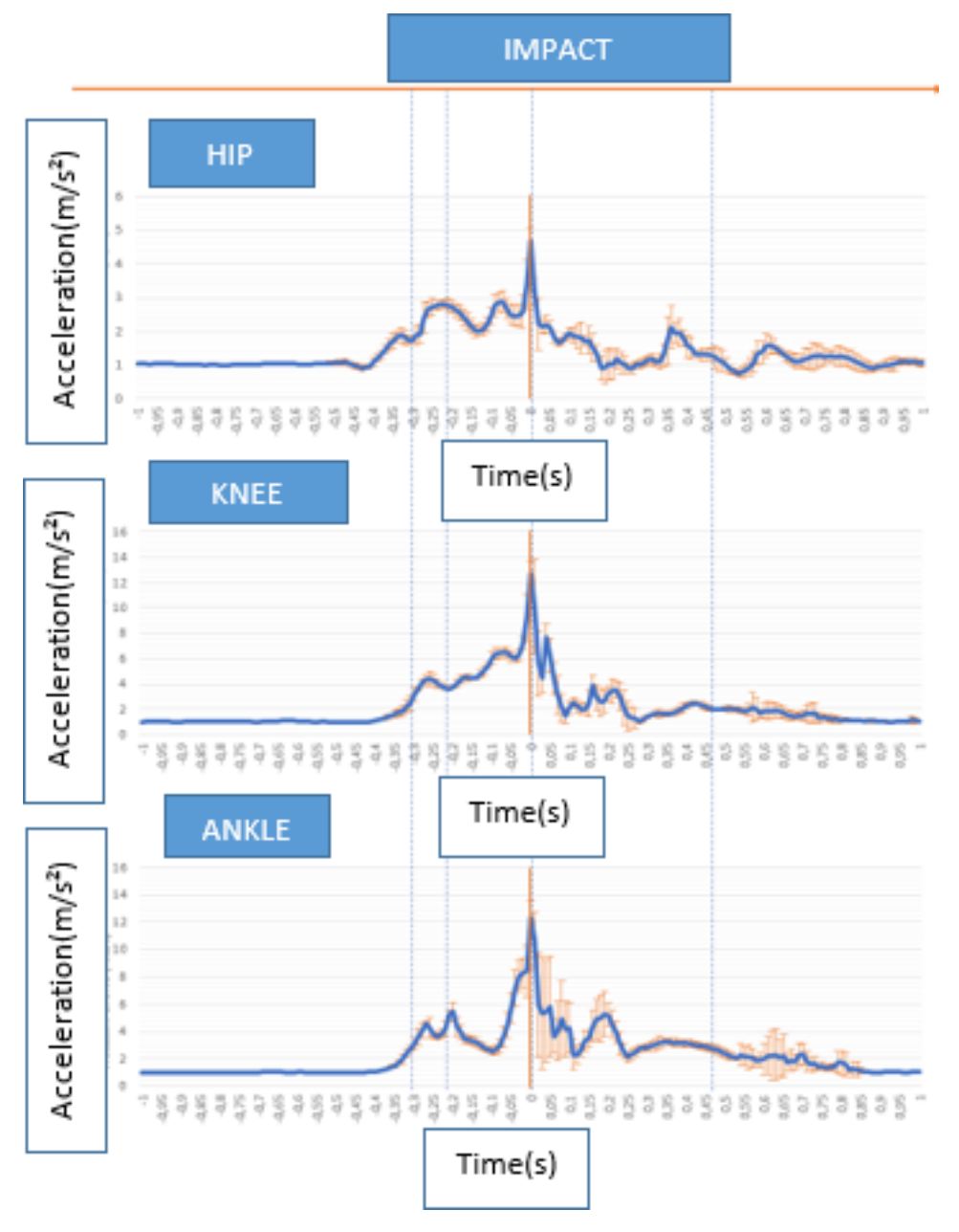

Figure 7. Acceleration values. 


\section{Phases of kick and angular velocity}

The figures of the average angular velocity for the three anatomical landmarks were aligned with the considerations on the motor task made by observing the frames from the videos. The result is visible in Table 5 . The values of the average angular velocity, in rad / s, of the seven kicks for each joint at the main events are described in the following table.

Table 5. Time angular speed values.

\begin{tabular}{lcccc}
\hline & Phase I & Phase II & Impact & Phase III \\
\hline Time & $-0.3 \mathrm{~s}$ & $-0.22 \mathrm{~s}$ & $0 \mathrm{~s}$ & $0.46 \mathrm{~s}$ \\
Hip (rad/s) & 175 & 333 & 179 & 182 \\
Knee (rad/s) & 151 & 257 & 826 & 195 \\
Ankle (rad/s) & 199 & 454 & 545 & 273 \\
\hline
\end{tabular}

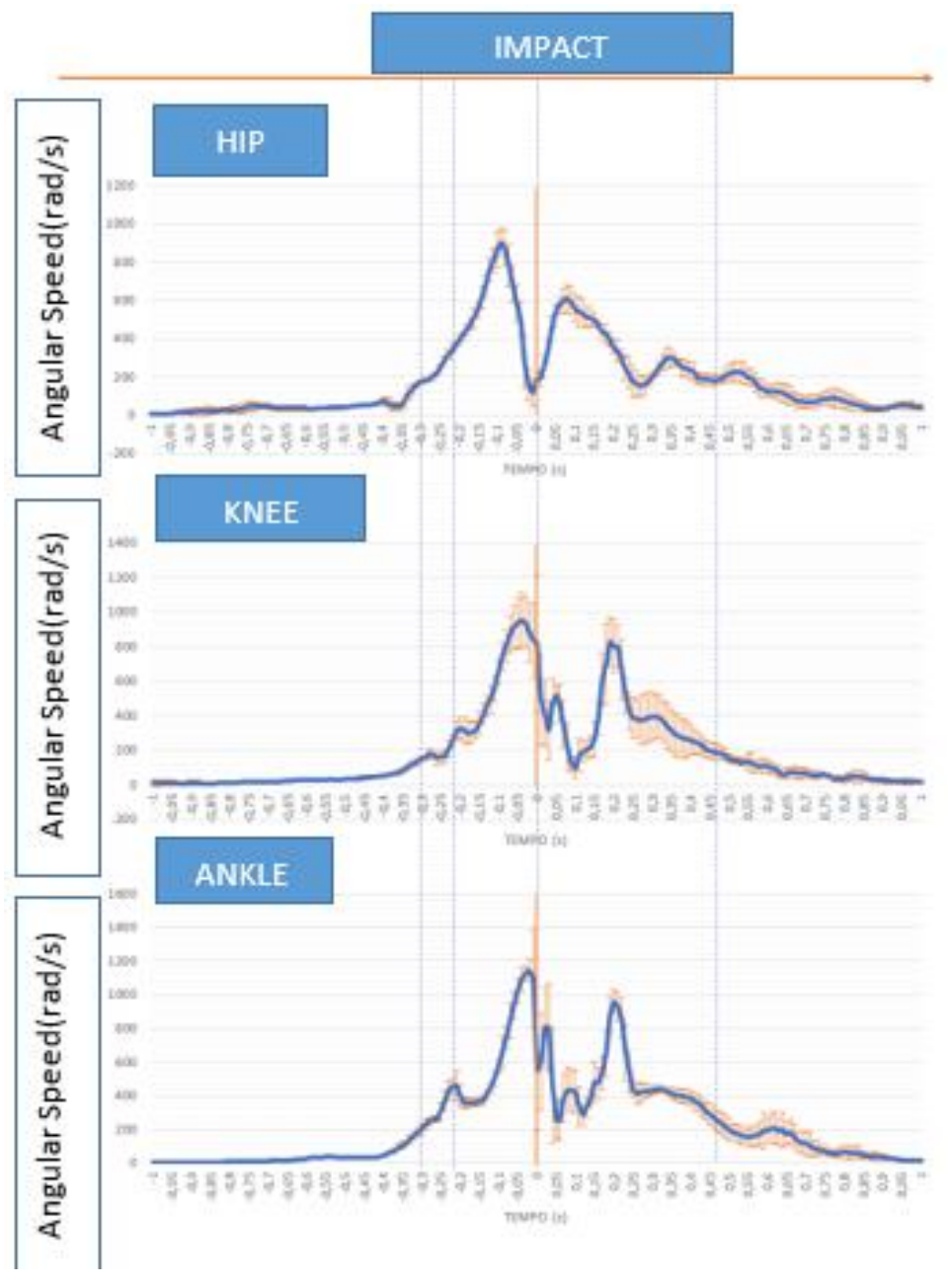

Figure 8. Angular speed values.

Observing the various phases, the following behaviors are noted:

- Preparation phase (I): there is an increasing increase in angular velocity in all three joints.

- Execution phase (II): The hip has a very strong angular acceleration for which the speed is increasing when the subject rotates $90^{\circ}$ counterclockwise; the knee and ankle have an increasing trend of 
angular velocity after which the latter decreases because there is no longer a rotation movement but becomes linear, in the moments preceding the impact. As regards the standard deviation measured between the samples under examination, it is noted that high values are recorded in the knee, probably due to the flexion-extension movement of the knee itself during execution.

- Return phase (III): In each joint there is an important peak of angular velocity, in the hip shortly after the impact being the first one that rotates it gives the push for the leg recall while for the knee and ankle this peak it is approximately 0.2 seconds after impact which represents the true rotational movement of the leg before the kicking foot lands. As for the standard deviation, also in this case it is greater in the knee probably for the same reason explained above.

\section{Impact strength considerations}

Having a measurement of the angular acceleration of the ankle at the moment of impact of the limb against the target allowed us to consider the force transferred to the bag. Strength is defined as:

$$
\mathrm{F}=\mathrm{m} \cdot \mathrm{a}
$$

Where $m$ represents the mass acting in $\mathrm{kg}$ and a its acceleration evaluated in $\mathrm{m} / \mathrm{s}^{2}$. In this case, for the calculation of the impact force, the acceleration of the ankle at instant $t=0$ was considered, while the following anatomical table was considered for the calculation of the active mass.
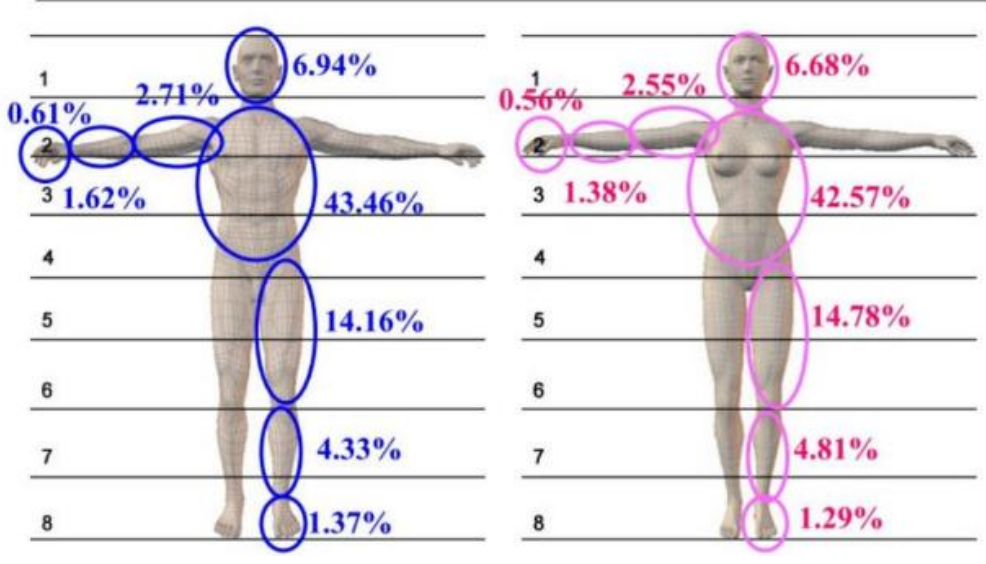

Figure 9. (https://ita.calameo.com)

It is evident that a leg has a mass about 4 times greater than an arm, so a leg strike and a punch strike will have the same momentum in the event that the punch technique has a speed four times that of the kick. and the same kinetic energy if the punch is twice as fast as the kick. In our case, the entire mass of the lower limb was considered as the acting mass. Therefore, considering the subject with a mass of $60 \mathrm{~kg}$, the mass in question will be equal to $12.53 \mathrm{~kg}$ (Figure 9). The acceleration of the ankle at the moment of impact, as previously written, is equal to $12.39 \mathrm{~m} / \mathrm{s} 2$. The impact force, taking these approximations into account, will therefore be equal to $155.25 \mathrm{~N}$.

\section{CONCLUSIONS}

In this study on the average circular kick in Muay Thai, the inertial sensor was used to evaluate the linear acceleration and angular velocity trend over time of three fundamental anatomical landmarks of the lower 
limb: hip, knee, and ankle. The analysis was initially conducted by observing the recordings made during the test from which fundamental events of the football motor task were identified, from which three specific phases were subsequently defined: preparation phase, execution phase and return phase. The values of the acceleration and angular velocity over time were then detected and then accompanied, in sync, to the analysis made on the frames and identify the acceleration and angular velocity values in the main instants. Following the considerations concerning the kinematics of football by observing the figures obtained for each reference point taken into consideration. Preparation phase, i.e., the taking of the loading of the leg; execution phase, i.e., the beginning of the athletic gesture; return phase, i.e., the support of the foot. In the preparation phase there is an increase in hip acceleration; the knee and ankle have a point of maximum acceleration at the same instant of time which corresponds to the load taking movement. In the same phase the angular velocity shows an increasing increase of angular velocity in all three joints. In the execution phase, the knee exhibits an increasing acceleration probably as a preparation for impact and therefore contact. The acceleration peak of the hip has a much lower value than the knee and ankle which, on the contrary, record similar values. The hip has a very strong angular acceleration which indicates an increase in speed. The knee and ankle have an increasing trend and then decrease because there is no more rotation movement, but it becomes linear in the moments preceding the impact. In the return phase, the acceleration decreases in all joints. In each joint there is an important peak of angular velocity, in the hip just before impact being the first joint that rotates and gives the push for the leg recall while for the knee and the ankle this peak is located at 0.2 seconds after the impact which represents the true rotational movement of the leg before the kicking foot lands. Finally, considerations on the force of impact were also made thanks to the processing of data from the applied instrument. Obviously, as mentioned, the study presented on a single athlete has no claim to evidence on the subject matter, for clear reasons of insufficient numbers of the sample from a statistical point of view, but the focus of the research was essentially to establish whether and how much the inertial technology of the latest generation used was applicable and what results could be useful for crossing elements of qualification and optimization of Muay Thai training in a performative function (Di Domenico et al., 2019a,b). It is clear that all of this was very effective, allowing us to establish a kinematic "wave" of the technical gesture that allowed an analysis of the moments as qualifying the success and its quality. It seems to us that this will allow the technical staff to rework the exercises on the same technical gesture with an activity of selection of the same elements strongly connected to the trend of the average circular football (Raiola et al., 2013, 2017). Given the possibility of carrying out these measurements even in live, which we will put in place in a short time, we can see the further chance of making changes in real time of the gesture performed by the athlete in training, without having to wait for the results of the download of the tool which would slow down training correction and in any case would not have the effect of a live application of technical information. Possible future developments will clearly include the expansion of the study sample to more subjects of both sexes and hopefully of different ages, both for considerations regarding the momentum as well as for energy aspects such as metabolic power and energy cost as a function of quality physical of the gesture (Di Domenico et al., 2020). In addition, the evaluation of the difference in kinematic terms between experienced subjects and beginners or amateurs will be of great interest, also relating the intervention, on the angles and joint trajectories, to the lower limb in relation to the sharing of the upper limb (Altavilla et al., 2020, Elia et al., 2020).

\section{REFERENCES}

Altavilla, G. (2019b). Monitoring training to adequate the teaching method in training: An interpretative concepts, Journal of Physical Education and Sport, 19, art. no. 258, pp. 1763-1766.

Altavilla, G. (2020). Energetic cost in the different running conditions in team sport for the educational teaching method, Sport Science, 14 (1), pp. 17-20. 
Altavilla, G., Mazzeo, F., D'Elia, F., Raiola, G. (2018). Physical commitment and specific work for each role in an elite soccer team, Journal of Physical Education and Sport, 18 (2), art. no. 83, pp. 570 574.

Altavilla, G., Raiola, G. (2019a). A brief review on physiological commitment in basketball: An interpretative key, Journal of Human Sport and Exercise, 14, pp. S59-S65. https://doi.org/10.14198/ihse.2019.14.Proc1.07

Baldassare G. (2012). Ricerca e didattica: analisi del movimento e nuove tecnologie, Guida Editore s.r.l.

Bos W., Favero S., Giannerini P. (1993) Taekwondo Tecniche di base e combattimento, Ed. Mediterranee.

Camillini A., D'Amen G., Izzo R. (2018). Concurrent training in the conditional planning of elite rugby player, International Scientific Conference: Perspectives in Physical Education and Sport, Ovidius University, 18th Edition, Constanta, Romania, 17-28.

Camomilla, V., Bergamini, E., Fantozzi, S., \& Vannozzi, G. (2018). Trends Supporting the In-Field Use of Wearable Inertial Sensors for Sport Performance Evaluation: A Systematic Review. Sensors, 18(3), 873. https://doi.org/10.3390/s18030873

Chinnasse C. et al. (2017) Lower limb kinematics kinematics analysis during roundhouse kick among novices in Muay Thai, Journal of Fundamental and Applied Science. 9(6S): p. 1002-1010. https://doi.org/10.4314/jfas.v9i6s.73

Crisafulli A., Vitelli S., Cappai I., Milia R., Tocco F., Mlis F.,Concu A. (2009) Fisiological responses and energy cost during a simulation of a Muay Thai boxing match, Appl. Physiol. Nutr. Metab. 34, 14350. https://doi.org/10.1139/H09-002

Cullane K., O'Connor M. ,Lyons D.,Lyons G. (2005). Accelerometers in rehabilitation medicine for older adults. Agr Ageing.Nov;34(6):556-60. https://doi.org/10.1093/ageing/afi192

De Cesaris M. (2009) Boxe Thailandese.Muay Thai, Roma, Ed. Mediterranee.

Del Vecchio FB., Silva JJR., Farias CB. (2015) Temporal analysis of national level Muay-Thai matches: Effects of competetive phases. Rev. Artes Marciales Asiàticas ,10, 34-41. https://doi.org/10.18002/rama.v10i1.1635

Di Domenico, F. (2020) From biomechanics to learning: Continuum for the theory of physical and sports education, Journal of Human Sport and Exercise, 15 (Proc2), pp. S268-S278. https://doi.org/10.14198/ihse.2020.15.Proc2.18

Di Domenico, F., D'isanto, T., Raiola, G. (2019b). Role of speed and agility in the effectiveness of motor performance, Journal of Physical Education and Sport, 19, art. no. 271, pp. 1836-1842.

Di Domenico, F., Fattore, S., Pignato, S., D'isanto, T. (2019a). Relationship between motor learning and reaction capacity in motor task, Journal of Human Sport and Exercise, 14 (Proc4), pp. S1030-S1037. https://doi.org/10.14198/jhse.2019.14.Proc4.65

Di Stefano R. (2004) Psiche marziale: arti del combattimento per la crescita psicofisica, Edizioni Mediterranee.

D'Isanto, T., D'Elia, F., Raiola, G., Altavilla, G. (2019). Assessment of sport performance: Theoretical aspects and practical indications, Sport Mont, 17 (1), pp. 79-82. https://doi.org/10.26773/smj.190214

Elia, F.D., Domenico, F.D., Isanto, T.D., Altavilla, G., Raiola, G. (2020). From biomechanics to motor learning, Acta Medica Mediterranea, 36 (5), pp. 3073-3078.

Garavan J. Colin et al. (2017) A biomechanical analysis of the roundhouse kicking technique of expert practitioners: A comparison between the martial arts disciplines of Muay Thai, Karate, and Taekwondo, School of Sport and Healt Sciences, University of the Sunshine Coast, Maroochydore, Queensland, Australia. https://doi.org/10.1371/journal.pone.0182645

Halil T., Fatma A., Metin K., Cecilia G., Mehemet K., Nurtekin E. (2010) Examination of the injures on the Muay Thai athletes, Ovidius Univ. Ann. Ser. Phys. Educ. Sport Mov. Heal, 10, 197-202. 
Hori N., Robert M. Newton U., Kazunori Nosaka, PhD (2005) Edith Cowan University, Joondalup, Western Australia, Weightlifting Exercises enhance athletic performance- that requires high-load speed strength, National Strength and Conditioning Association,Vol. 27,4,50-55. https://doi.org/10.1519/00126548-200508000-00008

Izzo R., Hosseini Varde'i C. (2018) Experimental approach via three different protocols on the speed agility in basketball: a case study, Journal of Physical Education and Sport (JPES), 18(2), Art 93, pp. 637-640.

Izzo R., Palpacelli C. (2018) Elementi determinanti correlati alla più corretta costruzione del lavoro di allenamento e della performance nello sport. L'attentività Operativa valutata tramite l'Inattentional Blindness Paradigm nel Multisport, Formazione e Insegnamento, European Journal of Research on Education and Teaching, anno XVI-no.1, pgg. 289-303, Pensa Multimedia.

Izzo R., Sebastianelli M., Hosseini Verde'i Ciro. (2018) Balance as quality of motory-sports performance in a target evaluation between advanced technology /IMU, International Journal of Physical Education, Sport and Healt, IJPESH; Vol. 4, issue 3, Impact Factor (ISRA) : 5.38, pag. 7-11, Tirupati J. Serv. Rohini, New Delhi, India.

Izzo R., Sopranzetti Seila, Molitierno Giulia. (2018) Video tracking for the detection of external load to establish proper parameters in elite soccer players during high intensity training, Journal of Physical Education and Sport (JPES), 18 Supplement issue 2, Art 178, pp. 1199-1202.

Izzo R., Vezzaro E., Hosseini Varde'i C. (2018) Quality study of some technical elements in artistic roller skating with use of new technologies, International Journal of Physical Education, Sport and Health, IJPESH; Vol. 5, issue 2, Impact Factor (ISRA): 5.38, pagg. 10-14, Tirupati J. Serv. Rohini, New Delhi, India.

Kim JW., Kwon MS., Yenuga S.S.\& Kwon YH. (2010) The effects of target distance on pivot hip,trunk, pelvis, and kicking leg kinematics in Taekwondo round house kick. Sports Biomechanics, 9,98-114. https://doi.org/10.1080/14763141003799459

Kyrolainen H. Avela J., Mc Bride JM, Koskinen S., Andersen J. Sipila s. et al. (2005) Effects of power training on muscle structure and neuromuscular performance, Scand J Med Sci Sports 15: 58-64. https://doi.org/10.1111/j.1600-0838.2004.00390.x

Landeo R. (2007) Fast front kick: gaining insights beyond the data, XXII ISBS Symposium. Ouro PretoBrazil, 298-301.

Loong L.H. (2011) Cultural Myths: Unpacking the origins of Muay Thai, Journal of Asian martial arts, 20, Issue 3, 33-36.

Martorelli L. (2013) Preparazione atletica negli sport da combattimento e nelle arti marziali, Milano, Hoepli.

Mathie M., Coster A.,Lovell N.,Celler. (2004) Accelerometry: providing an integrated, practical method for long-term, ambulatory monitoring of human movement. Physiol. Meas.; 25(2):R1-20. https://doi.org/10.1088/0967-3334/25/2/R01

Mohamad et al. (2016) Ricerche scientifiche nate sullo sport del Muay Thai: una rassegna della letteratura. Walailak Journal of science and technology,14, 615-625.

Mohamad N., Chinnasee C., Hemapandha W., Vongjaturapat N, ,Makaje N., Ratanarojanakool P., Pimjan L. (2017) Walailak Journal of science and technology, 2017; 14(8):616-625.

Montoye H.J., Kemper H., Saris W., Washburn R. (1996) Measuring physical activity and energy expenditure, Human Kinetics, Champaign.

Nakayama. (1994) Dynamické karate, Nase vojsko, Praha, ISBN 80-206-0408-1.

Park Y.J. (1989) A Biomechanical Analysis of Taekwondo Front-Kicks. Unpublished Doctoral Thesis, University of Minnesota. 
Pearson J.N. (1997) Kinematics and Kinetics of the taekwondo turning kick, Dissertation of degree bachlor of Physical Education with Honour of the University of Otago, Dunedin, New Zeeland.

Pfau T., Witte T.,Wilson A. (2005) A method for deriving displacment data during cyclical movement using an inertial sensor, The Journal of Experimental Biology, 208, 2503- 2514. https://doi.org/10.1242/jeb.01658

Raiola, G. (2013). Body knowledge and motor skills, Knowledge Cultures, 1 (6), pp. 64-72.

Raiola, G. (2017). Motor learning and teaching method Journal of Physical Education and Sport, 17, art. no. 236, pp. 2239-2243.

Raiola, G., Domenico, F.D., Isanto, T.D., Altavilla, G., Elia, F.D. (2020). Biomechanics core, Acta Medica Mediterranea, 36 (5), pp. 3079-3083.

Roberts E.M.,Zernicke R.F., Youm Y., Huang T.C. (1994) Kinetic parameters of kicking. Paper presented at Biomechanics IV, University Park, P.A.

Roetenberg D. (2006) Inertial and Magnetic Sensing og Human Motion, Universitiy of Twente.

Sforza C., Turci M. Grassi G.P., Shirai Y.F., Pizzini G. (2002) Repetability of mae-geri keage in traditional karate: a three-dimensional analysis with black-belt karateka. Perceptual and motor skills, 95, 433444. https://doi.org/10.2466/pms.2002.95.2.433

Sidthilaw S. (1997) Kinetic e Kinematic Analysis of Thai Boxing roundhouse kicks. Unpublished doctoral thesis, University of Oregon.

Siesto M. (2007) Considerazioni sull'insegnamento delle Arti Marziali, kosmoclub.

Silva, J., Del Vecchio, F., Picanço, L., Takito, M., \& Franchini, E. (2011). Time-Motion analysis in MuayThai and Kick-Boxing amateur matches. Journal of Human Sport and Exercise, 6(3), 490-496. https://doi.org/10.4100/jhse.2011.63.02

Sona A.,Bertocco M. (2013) Introduzione alle misure elettroniche, LULU, second edition, 12-30.

Sorensen H., Zacho M., Simonsen E.B., Dyhre-Poulsen P., Klausen K. (1996) Dynamics of the martial arts high ftont kick, Journal of Sport Sciences;14, 483-495. https://doi.org/10.1080/02640419608727735

Turner A. (2009) Strength and conditioning for Muay Thai athletes, Strength Cond. Journal, 31, 78-92. https://doi.org/10.1519/SSC.0b013e3181b99603

Verkhoshansky Y. (1997) Mezzi e metodi per l'allenamento della forza esplosiva. Tutto sul metodo d'urto, Società Stampa Sportiva, ISBN 978-88-8313-079-3.

Witte K., Emmermacher P., Soren B., Potemberg J. (2007) Movement structures of round kicks in karate, XXII ISBS Symposium.Ouro Preto-Brazil.

Worsey M.T., Hugo G. Espinosa. J. B. Sheperd e D. V Thiel. (2019) Inertial sensors for performances Analysis in combat sports: a Systematic Review. https://doi.org/10.3390/sports7010028

Zvona, M., Kolarova K., Zahradnicek V.,Reguli Z., Vit M. (2012). Kinematic Analysis in Combative Sports. IDO Movement for Culture. Journal of Material Arts Anthropology, 12 (4), 12-19.

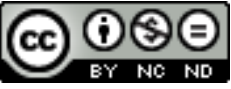

This work is licensed under a Attribution-NonCommercial-NoDerivatives 4.0 International (CC BY-NC-ND 4.0). 